Submitted to the Annals of Applied Statistics

\title{
SUPPLEMENTARY MATERIAL FOR 'A LOCALLY ADAPTIVE PROCESS-CONVOLUTION MODEL FOR ESTIMATING THE HEALTH IMPACT OF AIR POLLUTION'
}

\author{
By Duncan LeE ${ }^{\dagger}$ \\ University of Glasgow ${ }^{\dagger}$
}

Introduction. This supplementary material contains a number of different sections. Section 1 contains additional data summaries not included in the main paper, while Section 2 describes the pollution data modelling. Section 3 presents exploratory analysis of the likely correlation structures in the data to motivate the methodological development, while Section 4 presents the software used to fit the model. Section 5 derives the correlation structures implied to the process-convolution model, while Section 6 presents a sensitivity analysis of the results to model choice.

1. Additional data summaries. The locations of the $k=939$ GP surgeries coloured by health board is displayed in Figure 1, while a summary of the number of surgeries by health board is presented in Table 1 . The health boards with the largest numbers of GP surgeries are Greater Glasgow and Clyde (241) and Lothian (120), which contain the two largest cities Glasgow and Edinburgh and have populations of around 1.2 million and 0.85 million respectively. We consider respiratory prescriptions for medications that both prevent and relieve the symptoms of asthma and COPD. Medication that prevents the symptoms of asthma contain corticosteroids, while those that relieve symptoms contain short acting $\beta_{2}$ agonists. Details of the exact medications included in each group are given in Table 2 below.

A summary of the distributions of the variables in the study, including the standardised prescription rates (SPR), is given by Table 3, and shows some extreme high values as well as some GP surgeries with zero prescriptions for some months. The monthly temporal patterns in SPR for both medication types are displayed in Figure 2, and show little temporal variation except for December, which has a noticeably higher rate than the other months. Finally, the standardised prescription rates (SPR) for the two medication types are positively correlated with a Pearson's correlation coefficient of 0.60 , and a scatter plot of their values is shown in Figure 3.

\footnotetext{
${ }^{\dagger}$ Address for correspondence - Duncan.Lee@glasgow.ac.uk.
} 


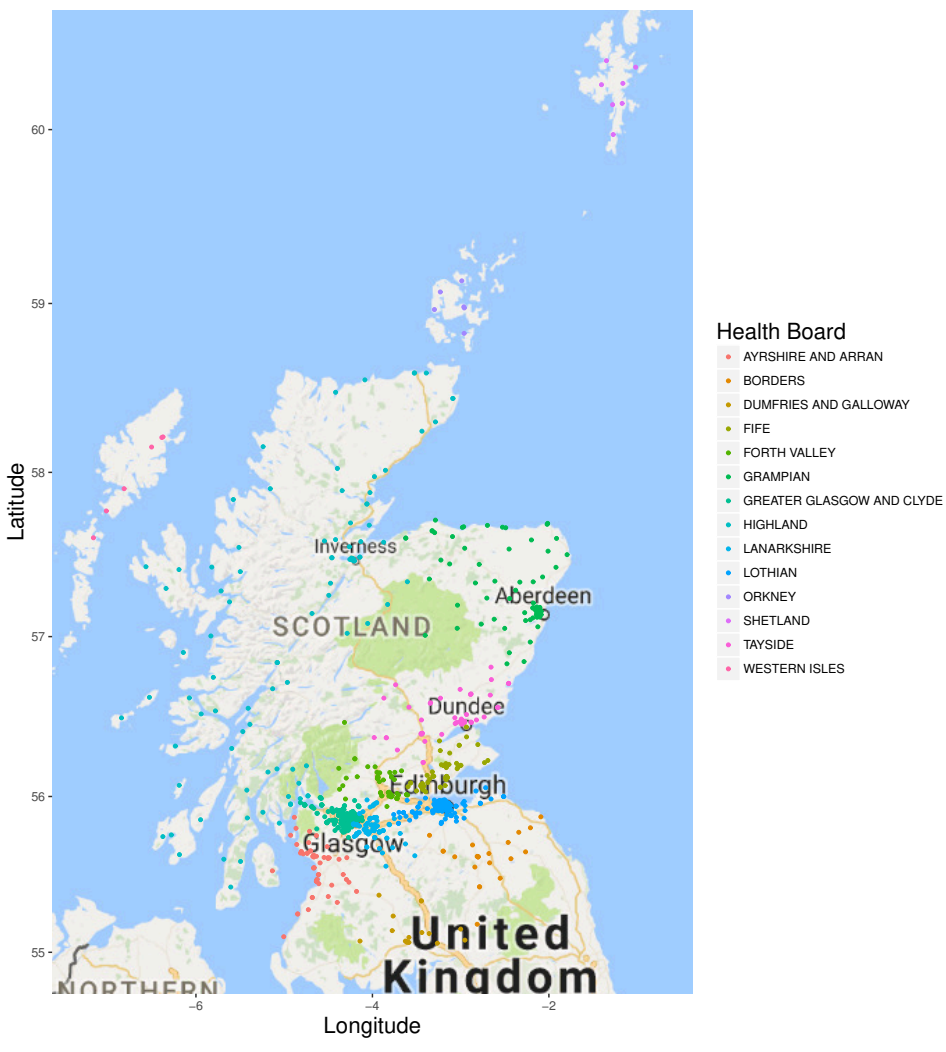

FIG 1. Map of Scotland showing the distribution of GP surgeries. The colours of the points indicate the Health Board each surgery is in.

TABLE 1

The number of GP surgeries in each Health Board.

\begin{tabular}{ll}
\hline Health Board & Number of GP surgeries \\
\hline Ayrshire and Arran & 55 \\
Borders & 23 \\
Dumfries and Galloway & 32 \\
Fife & 54 \\
Forth Valley & 54 \\
Grampian & 74 \\
Greater Glasgow and Clyde & 239 \\
Highland & 96 \\
Lanarkshire & 103 \\
Lothian & 120 \\
Orkney & 6 \\
Shetland & 10 \\
Tayside & 64 \\
Western Isles & 9 \\
\hline
\end{tabular}


TABle 2

Medications included in each category of respiratory medication together with their British National Formulary (BNF) codes.

\begin{tabular}{ll}
\hline Name & BNF code \\
\hline Reliever medication & \\
Salbutamol 100mcg & 0301011R0AAAPAP \\
Salbutamol $200 \mathrm{mcg}$ & 0301011R0AABXBX \\
Ventolin 100mcg & 0301011R0BEAIAP \\
Ventolin 200mcg & 0301011R0BEAHAQ \\
\hline Preventer medication & \\
Alvesco 80mcg & 0302000U0BBAAAA \\
Clenil Modulite 100mcg & 0302000C0BPABBF \\
Clenil Modulite 200mcg & 0302000C0BPACBV \\
Easyhaler Budesonide 200mcg & 0302000K0BGABAX \\
Flixotide Accuhaler 50mcg & 0302000N0BBARAR \\
Flixotide Accuhaler 250mcg & 0302000N0BBATAT \\
Flixotide Evohaler 50mcg & 0302000N0BBBBBH \\
Flixotide Evohaler 125mcg & 0302000N0BBAXBA \\
Flixotide Evohaler 250mcg & 0302000N0BBAZBC \\
Qvar 100mcg & 0302000C0BJABBF \\
\hline
\end{tabular}

TABle 3

Summary of the distribution of the non-categorical variables in the study.

\begin{tabular}{llrrrr}
\hline \multirow{2}{*}{ Variable } & \multicolumn{5}{c}{ Percentile of the distribution } \\
& $\mathbf{0 \%}$ & $\mathbf{2 5 \%}$ & $\mathbf{5 0 \%}$ & $\mathbf{7 5 \%}$ & $\mathbf{1 0 0 \%}$ \\
\hline Preventer prescriptions - counts & 0 & 20 & 33 & 50 & 224 \\
Preventer prescriptions - SPR & 0.00 & 0.75 & 0.98 & 1.27 & 5.14 \\
Reliever prescriptions - counts & 0 & 86 & 142 & 211 & 772 \\
Reliever prescriptions - SPR & 0.00 & 0.76 & 0.98 & 1.26 & 3.82 \\
\hline $\mathrm{PM}_{2.5}\left(\mu \mathrm{gm}^{-3}\right)$ & 4.36 & 7.06 & 7.87 & 8.49 & 16.52 \\
$\mathrm{PM}_{10}\left(\mu \mathrm{gm}^{-3}\right)$ & 6.17 & 9.87 & 11.06 & 12.07 & 23.39 \\
\hline percentage deprived & 0.00 & 0.07 & 8.13 & 26.34 & 87.04 \\
Property price $(£ 000)$ & 34.00 & 77.00 & 105.81 & 147.50 & 375.01 \\
\hline
\end{tabular}



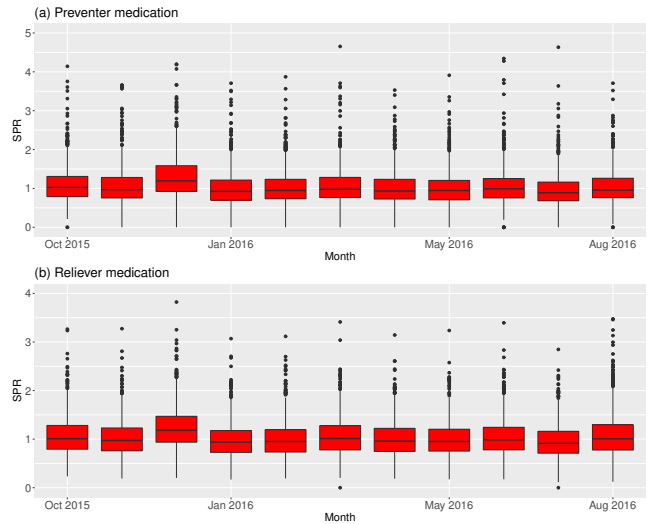

FIG 2. Boxplots showing the monthly variation in the SPR distribution for (a) preventer medication and (b) reliever medication. The black points illustrate the tails of the distribution, and are more extreme than 1.5 times the interquartile range from the lower or upper quartile.

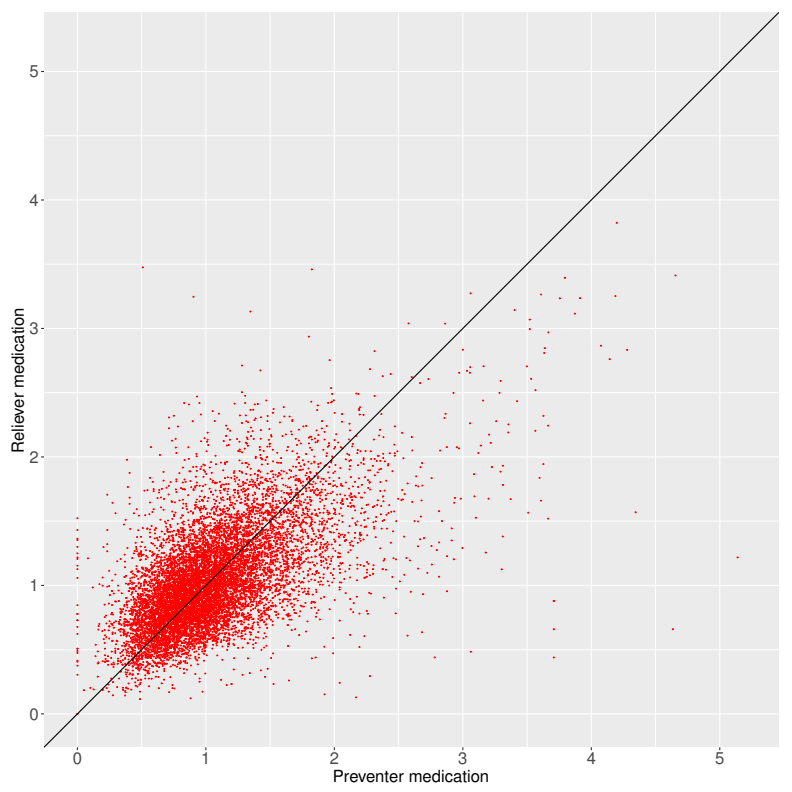

FIG 3. Scatterplot showing the relationship between the SPR values for preventer and reliever medication. 
2. Pollution data and modelling. The measured pollution concentrations are available at single point locations, numbering 76 sites for $\mathrm{PM}_{10}$ and 17 sites for $\mathrm{PM}_{2.5}$, although there were $6.7 \%$ and $7.2 \%$ missing values respectively for each pollutant over the 11 months. The locations of these sites for $\mathrm{PM}_{10}$ are displayed in Figure 4, where the local environment, roadside or background, in which the sites are located is illustrated by the colours of the data points. The 17 sites for $\mathrm{PM}_{2.5}$ form a subset of these 76 sites. The figure shows the spatial locations are clustered in the cities including Glasgow and Edinburgh, with large areas of northern and southern Scotland having no monitoring sites at all. Therefore we also use modelled annual average concentrations on a 1 kilometre grid provided by DEFRA for both pollutants, further details of which are provided by Steadman (2015). These modelled concentrations provide complete spatial coverage of Scotland, but are yearly rather than monthly averages.

To link particulate air pollution to the respiratory prescription data we need to estimate monthly average concentrations for each GP surgery, using both measured and modelled data sets via a simple fusion model. Specifically, we fit a series of normal additive regression models for each pollutant separately $\left(\mathrm{PM}_{2.5}\right.$ and $\left.\mathrm{PM}_{10}\right)$, where the response variable is the square root (a common transformation used when modelling air pollution, see e.g. Lee et al., 2017) of the monthly mean measured concentration for each site (17 for $\mathrm{PM}_{2.5}$ and 76 for $\mathrm{PM}_{10}$ ) and month. Covariates considered in the model include the square root of the nearest modelled grid level concentration (denoted DEFRA), a factor variable for month (denoted month), a factor variable for the site type of the measured concentration (roadside or background, denoted site), and geographical location to account for spatial variation (denoted easting, northing). We consider a variety of different models, the most complex of which exhibit spatially varying intercepts and slopes (for the modelled concentrations), which follows the work of Berrocal et al. (2010).

The predictive performances of the models are compared via a 10-fold cross validation exercise, which was conducted separately for $\mathrm{PM}_{10}$ and $\mathrm{PM}_{2.5}$. The cross validation was repeated 10 times with different random groupings, which will reduce the random variation in the results due to group choice. In each run of this cross-validation exercise the $76 \mathrm{PM}_{10}$ or 17 $\mathrm{PM}_{2.5}$ sites are split into 10 near equal sized groups (as the numbers of sites do not divide exactly by 10) at random. Then each model is fitted to data from 9 out of 10 of these groups, before being used to predict the pollution concentrations from the 10th (prediction) group. This process is iterated 10 times so that each group becomes the prediction group in turn. 
We note that each site is in the prediction set for all 11 months, which mimics the real life situation of having to predict pollution where there has not been a site at any point during the study period. Additionally, we note that cross validation for spatio-temporal data can be affected by the presence of residual spatio-temporal correlations after covariate adjustment, which affects the desirable approach to choosing the spatial locations of the fitting and prediction data sets (see Roberts et al., 2017 for a recent review). However, here the residuals from even the simplest model (including grid level concentrations and factors for month and site type) are independent, which was assessed by variogram (spatial) and autocorrelation and partial autocorrelation (both temporal) analysis. Therefore we utilise a random partition of the pollution monitor sites in each run of the cross validation exercise.

The complete set of predictions from each model are jointly summarised in Table 4. Specifically, the table displays the predictive bias and the root mean square prediction error (RMSPE) for a range of models for both pollutants, where all results have been back transformed to the original scale. The first row of the table is based on predicting each monitor location by the closest gridded observation (same for each month and not from a model), and offers a baseline comparison. In the table $f a c()$ denotes that a variable was modelled as a factor, while $\mathbf{s}()$ denotes it was modelled as a smooth function within the gam () function in the mgcv package. Finally, a + denotes an additive effect while a $*$ denotes an interaction (with main effects) between two variables.

The table shows that firstly most of the fusion models have better predictive performance than the raw DEFRA data, with smaller bias and RMSPE values. Second, comparing rows 2 and 3 shows that treating month as a factor variable outperformed treating it as continuous smooth function. Third, Rows 4 and 5 show that additionally including an interaction between the modelled concentrations and either month or site type reduced the predictive performance, suggesting that the effect of the DEFRA modelled data should be constant over time and over site type. Finally, rows 6 and 7 add in a spatially varying intercept (row 6) and slope (row 7) for the DEFRA modelled concentrations (via a smooth function of easting and northing), and both lead to poorer predictive performance. The simple additive model (row 2) was the best performing fusion model for both pollutants, and as mentioned above empirical semi-variogram and sample autocorrelation analysis showed its residuals showed no evidence of any spatio-temporal autocorrelation. Thus monthly predictions were made from the simple additive model at each GP location and month, where in all cases the site type for the predictions were set to background rather than roadside to be more representative 


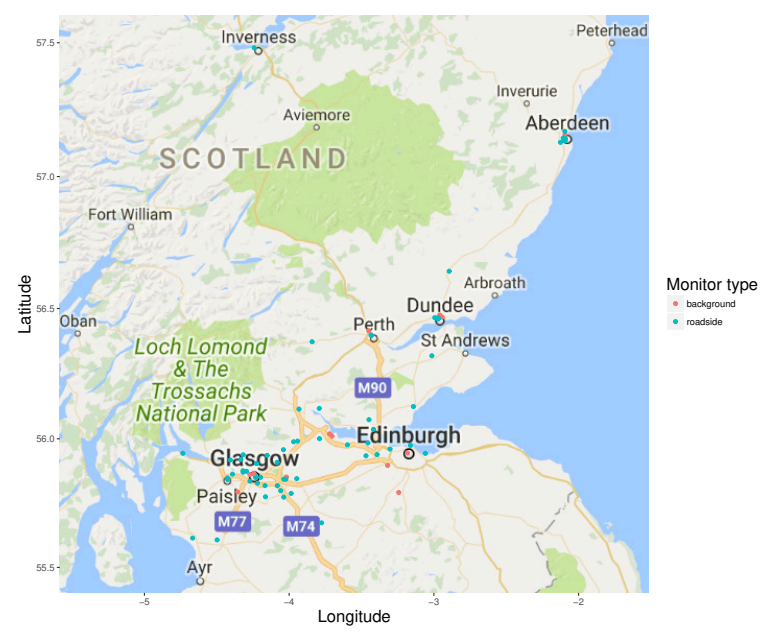

FIG 4. Locations of the pollution monitors in Scotland measuring $P M_{10}$.

TABLE 4

Results from the ten-fold cross validation exercise for both pollutants.

\begin{tabular}{lllll}
\hline \multirow{2}{*}{ Model } & \multicolumn{2}{c}{ PM $_{10}$} & \multicolumn{2}{c}{ PM $_{2.5}$} \\
& Bias & RMSPE & Bias & RMSPE \\
\hline DEFRA & -3.294 & 5.112 & 0.734 & 2.211 \\
fac (Month) + fac(site) + DEFRA & -0.192 & 3.325 & -0.038 & 1.784 \\
s(Month) + fac(site) + DEFRA & -0.192 & 3.336 & -0.038 & 1.819 \\
fac (Month) + fac(site) * DEFRA & -0.180 & 3.363 & 0.413 & 2.664 \\
fac (Month) * DEFRA + fac(site) & -0.186 & 3.356 & -0.032 & 1.797 \\
fac(Month) + DEFRA + fac(site) + s(easting, northing) & 0.207 & 4.498 & -0.144 & 2.283 \\
fac(Month) + fac(site) + s(easting, northing) * DEFRA & 0.157 & 4.496 & -0.056 & 2.380 \\
\hline
\end{tabular}

of population exposure.

3. Exploratory data analysis. Initially, an overdispersed Poisson loglinear model was fitted to the prescription count data for each medication type (preventer or reliever) separately, so that the presence of residual spatiotemporal autocorrelation could be investigated. The covariates included the expected numbers of prescriptions (as a fixed offset), $\mathrm{PM}_{2.5}$ concentrations, deprivation based on SIMD, an indicator variable for December, and a factor variable for health board. The residuals from this model were then investigated for the presence and type of spatio-temporal and between medication (preventer vs reliever) correlations. The between medication correlations in the residuals (over space) were very similar over time, ranging between 0.50 

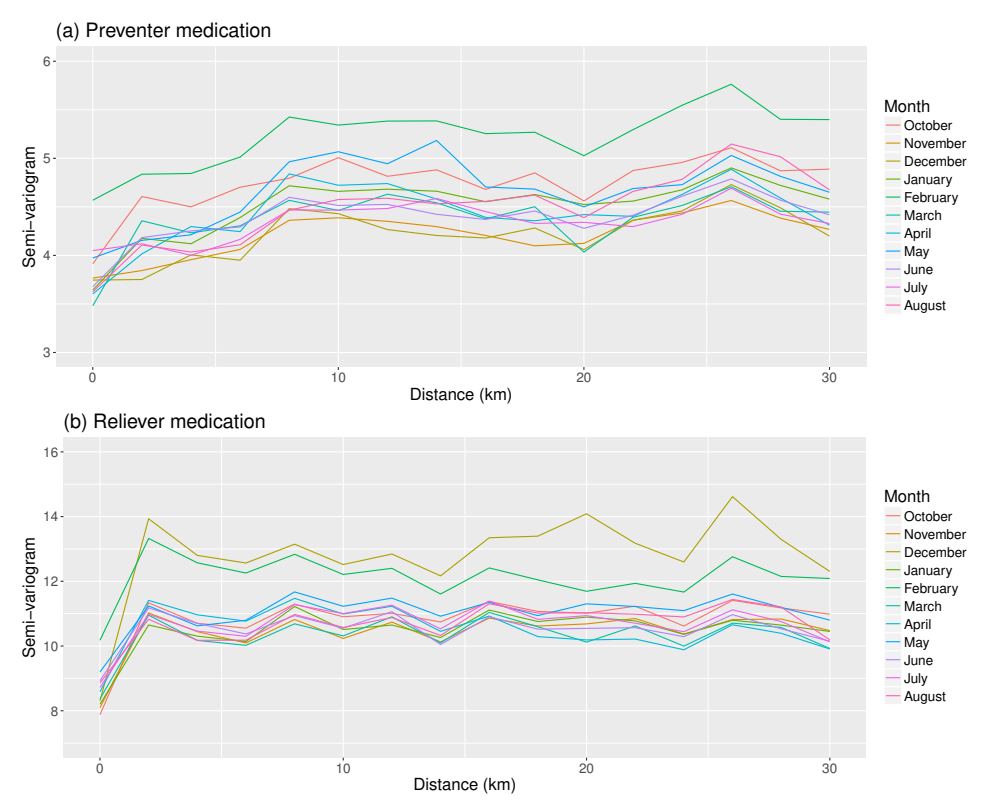

FIG 5. Empirical variograms of the residuals from a simple Poisson log-linear model estimated separately for each month for (a) preventer and (b) reliever medication.

and 0.59 across the 11 months, suggesting that assuming there is a single between medication correlation is reasonable for these data, that is thus separable from the spatio-temporal correlation structure. Temporal correlation is difficult to assess for these data given there are only 11 time periods, so in the methodology that follows we include a parameter to control the strength of the temporal correlation in the model.

Empirical variograms were then computed for the residuals from each medication type separately for each year, and are displayed in Figure 5. The figure shows the presence of short-range spatial correlation in the residuals, particularly evident for reliever medication, suggesting that an additional spatially correlated process should be included in the model to account for this. Figure 5 also shows that the variograms from the different months are similar, relative to the uncertainty in variogram estimation, suggesting that a separable spatio-temporal covariance structure will be appropriate for these data.

4. Model fitting, computer software and data. The general model defined by equations (3.1) - (3.2) and with weights defined by (3.3) or (3.4) is fitted in a Bayesian framework via Markov chain Monte Carlo (MCMC) simulation, and uses a combination of Gibbs sampling and Metropolis-Hastings 
updating steps. The parameters controlling the temporal autocorrelation $(\gamma)$ check........ and the between medication covariance matrix $(\Sigma)$ are Gibbs sampled, because their full conditional distributions are Gaussian and Inverse-Wishart respectively. The remaining parameters do not have full conditional distributions that are standard forms, and hence are updated using Metropolis or Metropolis-Hastings steps. Specifically, the regression parameters are updated using the Metropolis Adjusted Langevin Algorithm (MALA, Roberts and Rosenthal, 1998) in blocks of size 10, which exploratory analysis showed performed better than a simple random walk step. The latent process at location $\mathbf{s}_{k}$ and month $t, \boldsymbol{\theta}_{t}\left(\mathbf{s}_{k}\right)=\left(\theta_{t 1}\left(\mathbf{s}_{k}\right), \theta_{t 2}\left(\mathbf{s}_{k}\right)\right)$, is updated separately for each above block of two parameters using a random walk Metropolis step. Finally, for model $(3.4) \boldsymbol{\psi}_{k}=\left(\psi_{k 1}, \ldots, \psi_{k m}\right)$ is updated in a block of size $m$ separately for each $k$ using random walk proposals, while for model (3.3) $\boldsymbol{\delta}$ is updated jointly using random walk proposals.

Software to fit the model is written in the $\mathrm{R}$ ( $\mathrm{R}$ Core Team, 2016) programming language, and is made computationally efficient by the use of C++ subroutines written using the Rcpp (Eddelbuettel and Francois, 2011) package, as well as taking advantage of the sparsity of $\mathbf{W}$ via its triplet form representation. To make this research reproducible both the data and the software are provided here, including the following files.

- PCkernel.R - function to fit the Kernel smoothing model given by (3.1), (3.2) and (3.3).

- PClocalised.R - function to fit the locally adaptive smoothing model given by (3.1), (3.2) and (3.4).

- PCST.cpp - C++ functions to sample parameters in both Kernel and localised weight models.

- common.functions. R - R helper functions.

- Run the analysis. $R$ - script file that reads in the data and fits both Kernel and locally adaptive weight models with $m=8$.

Also provided with this supplementary material is the data GPdata.csv, which contains the following columns.

- code - unique code of each GP surgery.

- month - the month the data relate to.

- prev - the number of prescriptions for preventer medication.

- reli - the number of prescriptions for reliever medication.

- board - health board the GP surgery resides in.

- easting - east-west geographical coordinate of the GP surgery.

- northing - north-south geographical coordinate of the GP surgery.

- dep.simd - the percentage of patients that live in the $15 \%$ most de- 
prived datazones as measured by the Scottish Index of Multiple Deprivation (SIMD).

- pm25 - estimated $\mathrm{PM}_{2.5}$ concentrations.

- Eprev - the expected number of prescriptions for preventer medications.

- Ereli - the expected number of prescriptions for reliever medications.

- SPRprev - the standardised prescription rate for preventer medications.

- SPRreli - the standardised prescription rate for reliever medications.

- urban - the level of urbanicity of the location of the GP surgery.

5. Correlation structure of the process-convolution. The correlation structure between two random effects $\left(\phi_{t j}\left(\mathbf{s}_{k}\right), \phi_{r l}\left(\mathbf{s}_{i}\right)\right)$ can be obtained using multivariate Gaussian theory as follows. First, let $\boldsymbol{\theta}\left(\mathbf{s}_{k}\right)=$ $\left(\boldsymbol{\theta}_{1}\left(\mathbf{s}_{k}\right), \ldots, \boldsymbol{\theta}_{N}\left(\mathbf{s}_{k}\right)\right)$, where $\boldsymbol{\theta}_{t}\left(\mathbf{s}_{k}\right)=\left(\theta_{t 1}\left(\mathbf{s}_{k}\right), \theta_{t 2}\left(\mathbf{s}_{k}\right)\right)$. Then $\boldsymbol{\theta}\left(\mathbf{s}_{k}\right)$ is modelled by the first-order autoregressive process given by (3.2) in the main paper, which can be re-written as $\boldsymbol{\theta}\left(\mathbf{s}_{k}\right) \sim \mathrm{N}\left(\mathbf{0},\left[\mathbf{Q} \otimes \boldsymbol{\Sigma}^{-1}\right]^{-1}\right)$, where $\mathbf{0}$ is an $2 N \times 1$ vector of zeros and $\otimes$ is the Kronecker product. The autoregressive precision matrix $\mathbf{Q}_{N \times N}$ is tridiagonal and given by:

$$
\mathbf{Q}=\left(\begin{array}{lllll}
1+\gamma^{2} & -\gamma & & & \\
-\gamma & 1+\gamma^{2} & -\gamma & & \\
& \ddots & \ddots & \ddots & \\
& & -\gamma & 1+\gamma^{2} & -\gamma \\
& & & -\gamma & 1
\end{array}\right)
$$

Then letting $\boldsymbol{\theta}=\left(\boldsymbol{\theta}\left(\mathbf{s}_{1}\right), \ldots, \boldsymbol{\theta}\left(\mathbf{s}_{K}\right)\right)$ it follows that

$$
\boldsymbol{\theta} \sim \mathrm{N}\left(\mathbf{0},\left[\mathbf{I}_{K} \otimes\left(\mathbf{Q} \otimes \boldsymbol{\Sigma}^{-1}\right)\right]^{-1}\right),
$$

where $\mathbf{I}_{K}$ is the $K \times K$ identity matrix. Then writing $\phi=\left(\phi\left(\mathbf{s}_{1}\right), \ldots, \phi\left(\mathbf{s}_{K}\right)\right)$, where $\boldsymbol{\phi}\left(\mathbf{s}_{k}\right)=\left(\phi_{1}\left(\mathbf{s}_{k}\right), \ldots, \phi_{N}\left(\mathbf{s}_{k}\right)\right)$, it follows that $\boldsymbol{\phi}=\left(\mathbf{W} \otimes \mathbf{I}_{2 N}\right) \boldsymbol{\theta}$, where $\mathbf{I}_{2 N}$ is the $2 N \times 2 N$ identity matrix. Then using (5.2) we have that:

$$
\begin{aligned}
\boldsymbol{\phi} & \sim \mathrm{N}\left(\mathbf{0},\left[\mathbf{W} \otimes \mathbf{I}_{2 N}\right]\left[\mathbf{I}_{K} \otimes\left(\mathbf{Q} \otimes \mathbf{\Sigma}^{-1}\right)\right]^{-1}\left[\mathbf{W} \otimes \mathbf{I}_{2 N}\right]^{\top}\right) \\
& \sim \mathrm{N}\left(\mathbf{0},\left[\mathbf{W} \mathbf{W}^{\top} \otimes\left(\mathbf{Q}^{-1} \otimes \mathbf{\Sigma}\right)\right]\right) .
\end{aligned}
$$

Thus $\mathbb{E}\left[\phi_{t i}\left(\mathbf{s}_{k}\right)\right]=0$ for all $(k, t, i)$, whilst the variance, between medication, temporal and spatial correlations simplify to: 


$$
\begin{aligned}
\operatorname{Var}\left[\phi_{t i}\left(\mathbf{s}_{k}\right)\right] & =\Sigma_{i i}\left[\mathbf{Q}^{-1}\right]_{t t} \sum_{j=1}^{K} w_{k j}^{2}, \\
\operatorname{Corr}\left[\phi_{t 1}\left(\mathbf{s}_{k}\right), \phi_{t 2}\left(\mathbf{s}_{k}\right)\right] & =\frac{\Sigma_{12}}{\sqrt{\Sigma_{11} \Sigma_{22}}}, \\
\operatorname{Corr}\left[\phi_{t i}\left(\mathbf{s}_{k}\right), \phi_{r i}\left(\mathbf{s}_{k}\right)\right] & =\frac{\left[\mathbf{Q}^{-1}\right]_{t r}}{\sqrt{\left[\mathbf{Q}^{-1}\right]_{t t}\left[\mathbf{Q}^{-1}\right]_{r r}}}, \\
\operatorname{Corr}\left[\phi_{t i}\left(\mathbf{s}_{k}\right), \phi_{t i}\left(\mathbf{s}_{l}\right)\right] & =\frac{\sum_{j=1}^{K} w_{k j} w_{l j}}{\sqrt{\left(\sum_{j=1}^{K} w_{k j}^{2}\right)\left(\sum_{j=1}^{K} w_{l j}^{2}\right)}} .
\end{aligned}
$$

The variance depends on the between medication structure via $\boldsymbol{\Sigma}$, the spatial structure via the weights $\mathbf{W}$ and the temporal structure via $\mathbf{Q}$. The between medication, spatial, and temporal correlation structures separate, in the sense that the between medication correlation just depends on $\boldsymbol{\Sigma}$, the temporal autocorrelation depends only on $\mathbf{Q}$, whilst the spatial autocorrelation depends only on $\mathbf{W}$. This separability is appropriate for these data following the exploratory analysis in Section 3.

6. Sensitivity analyses. Recall that we have two proxy measures of socio-economic deprivation, the percentage of patients from each GP surgery who live in the $15 \%$ most deprived datazones based on the Scottish Index of Multiple Deprivation (SIMD), and the median property price surrounding each GP surgery. The main paper presented the estimated pollution effects for the first metric, while here we contrast these results with those obtained by replacing that deprivation measure with median property price. The results presented here relate to $m=8$ from the localised model, and are as follows:

- $\mathrm{PM}_{2.5}$ - preventer medication - the estimated effects are 1.018 (1.013, 1.024) for the SIMD based measure of deprivation and 1.015 (1.010, 1.020) for the property price measure.

- $\mathrm{PM}_{2.5}$ - reliever medication - the estimated effects are 1.017 (1.013, 1.020) for the SIMD based measure of deprivation and 1.019 (1.015, 1.023) for the property price measure.

- $\mathrm{PM}_{10}$ - preventer medication - the estimated effects are 1.014 (1.011, 1.018) for the SIMD based measure of deprivation and 1.011 (1.008, 1.014) for the property price measure. 
- $\mathrm{PM}_{10}$ - reliever medication - the estimated effects are 1.011 (1.009, 1.014) for the SIMD based measure of deprivation and 1.013 (1.011, 1.016) for the property price measure.

The effect sizes differ slightly (max difference of $0.3 \%$ ) but the overall conclusions are unchanged, in that rates of medication for both preventer and reliever medication are associated with increased rates in both pollutants. 


\section{References.}

Berrocal, V., A. Gelfand, and D. Holland (2010). A spatio-temporal downscaler for output from numerical models. Journal of Agricultural, Biological and Environmental Statistics 15, 176-197.

Eddelbuettel, D. and R. Francois (2011). Rcpp: Seamless R and C++ Integration. Journal of Statistical Software 40, 1-18.

Lee, D., S. Mukhopadhyay, A. Rushworth, and S. Sahu (2017). A rigorous statistical framework for spatio-temporal pollution prediction and estimation of its long-term impact on health. Biostatistics 18, 370-385.

R Core Team (2016). R: A Language and Environment for Statistical Computing. Vienna, Austria: R Foundation for Statistical Computing.

Roberts, D., V. Bahn, S. Ciuti, M. Boyce, J. Elith, G. Guillera-Arroita, S. Hauenstein, J. Lahoz-Monfort, B. Shroder, W. Thuiller, D. Warton, B. Wintle, F. Hartig, and C. Dormann (2017). Cross-validation strategies for data with temporal, spatial, hierarchical, or phylogenetic structure. Ecography 40, 913-929.

Roberts, G. and J. Rosenthal (1998). Optimal scaling of discrete approximations to the Langevin diffusions. Journal of the Royal Statistical Society B 60, 255-268.

Steadman, J. (2015). Technical report on UK supplementary assessment under the Air Quality Directive (2008/50/ec), the Air Quality Framework Directive (96/62/ec) and Fourth Daughter Directive (2004/107/ec) for 2013. Technical report, Ricardo-AEA.

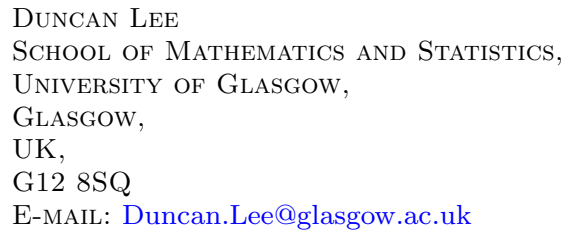

